\title{
Statistical evolution of isotope composition of nuclear fragments.
}

\author{
A.S. Botvina ${ }^{a, b}$ and I.N. Mishustin ${ }^{c, d}$ \\ ${ }^{a}$ Gesellschaft für Schwerionenforschung, D-64291 Darmstadt, Germany \\ ${ }^{b}$ Institute for Nuclear Research, 117312 Moscow, Russia \\ ${ }^{c}$ Niels Bohr Institute, DK-2100 Copenhagen, Denmark \\ ${ }^{d}$ Kurchatov Institute, Russian Research Center, 123182 Moscow, Russia
}

(October 28, 2018)

\begin{abstract}
Calculations within the statistical multifragmentation model show that the neutron content of intermediate mass fragments can increase in the region of liquid-gas phase transition in finite nuclei. The model predicts also inhomogeneous distributions of fragments and their isospin in the freeze-out volume caused by an angular momentum and external long-range Coulomb field. These effects can take place in peripheral nucleus-nucleus collisions at intermediate energies and lead to neutron-rich isotopes produced in the midrapidity kinematic region.
\end{abstract}

PACS number(s): 25.70.Pq, 24.10.Pa, 25.70.Mn 
Multifragmentation of nuclei is the most interesting phenomenon in intermediate energy nuclear physics. This is a promising process for studying nuclear matter properties at extreme conditions of high excitation energies, small densities and at different isospins. In particular, one hopes to establish its connection to a nuclear liquid-gas phase transition [1]. As other complicated many-body processes this phenomenon can be successfully treated in a statistical way [2,3]. Fragment production in both peripheral [4, [5] and central [6,7] collisions has clear statistical features, though a considerable preequilibrium emission and collective energy (e.g. radial flow) should be taken into consideration. However, in finite-size nuclear systems statistical processes can lead to specific effects since the fragment formation is governed by both short-range nuclear forces and long-range Coulomb forces. We show in this paper how the interplay of these different forces is manifested in isotope production. Also we show that an external Coulomb field, e.g. a Coulomb interaction of the target and projectile-like sources in peripheral nucleus-nucleus collisions, can provide a non-isotropic statistical emission of fragments and cause asymmetry in the fragment isospin distribution.

The statistical multifragmentation model (SMM) is described in detail in many publications, see e.g. [2]. The model is based upon the assumption of statistical equilibrium at a low-density freeze-out stage. We consider all break-up channels (partitions) composed of nucleons and excited fragments taking into account mass, charge, momentum and energy conservations. In the microcanonical treatment the statistical weight of decay channel $\mathrm{j}$ is given by $W_{j} \propto \exp S_{j}$, where $S_{j}$ is the entropy of the system in channel $j$ depending on excitation energy $E_{s}^{*}$, mass number $A_{s}$, charge $Z_{s}$ and other parameters of the source. Light fragments with mass number $A \leq 4$ are considered as stable particles ("nuclear gas") with only translational degrees of freedom; fragments with $A>4$ are treated as heated nuclear liquid drops. In the standard version [2] the Coulomb interaction between the fragments is treated in the Wigner-Seitz approximation. Different break-up partitions are sampled according to their statistical weights uniformly in the phase space. After break-up of the nuclear source the fragments propagate independently in their mutual Coulomb field and undergo secondary decays. The deexcitation of the hot primary fragments proceeds via 
evaporation, fission or Fermi-break-up [8].

A new version of SMM [9, [10] is based on generating the Markov chain of partitions which is representative of the whole partition ensemble [11]. Individual partitions are generated and selected into the chain by applying the Metropolis algorithm and taking into account that fragments with the same mass $A$ and charge $Z$ are indistinguishable. Due to a high efficiency of this method one can directly calculate the Coulomb interaction energy for each spatial configuration of primary fragments in the freeze-out volume. In this way one can take into account the correlations between positions of the primary fragments and their Coulomb energy that influences the partition probabilities [3]. Also one can calculate the moment of inertia and take into account the angular momentum conservation similar to Refs. [3].12]. The new method is consistent with the previous one [2] based on the Wigner-Seitz approximation. The mean parameters of produced fragments obtained in the standard and the Markov chain versions fit each other reasonably well. The full analysis of the Markov chain SMM appears elsewhere [10], here we present our new results concerning the isospin degree of freedom in nuclear multifragmentation and provide some guidelines for future studies. We concentrate on properties of hot primary fragments since, apart of very light fragments, the secondary decay doesn't change the predicted isospin evolution.

Before any application it is important to demonstrate that the Monte-Carlo generation of the Markov chain exactly represents the whole ensemble of fragment partitions. The case of one component system (that takes into account only nucleons without isospin) was considered in [9]. Here we present results for the two-component system when fragments are characterized by both the mass number and charge. The best way to check the numerical algorithm is to compare it with the direct calculation accounting for all possible partitions, one by one. As shown in [9], the precision of the Monte-Carlo algorithm is very good and does not depend significantly on the system's size. Fig. 1 shows the distributions of average fragment multiplicities and average charges versus mass number of fragments for two cases which differ by the partition weights. In the first case all partitions are taken with equal weights, while in the second case, with the factorial weights, $W=1 / \prod N_{A Z}$ !, where $N_{A Z}$ is 


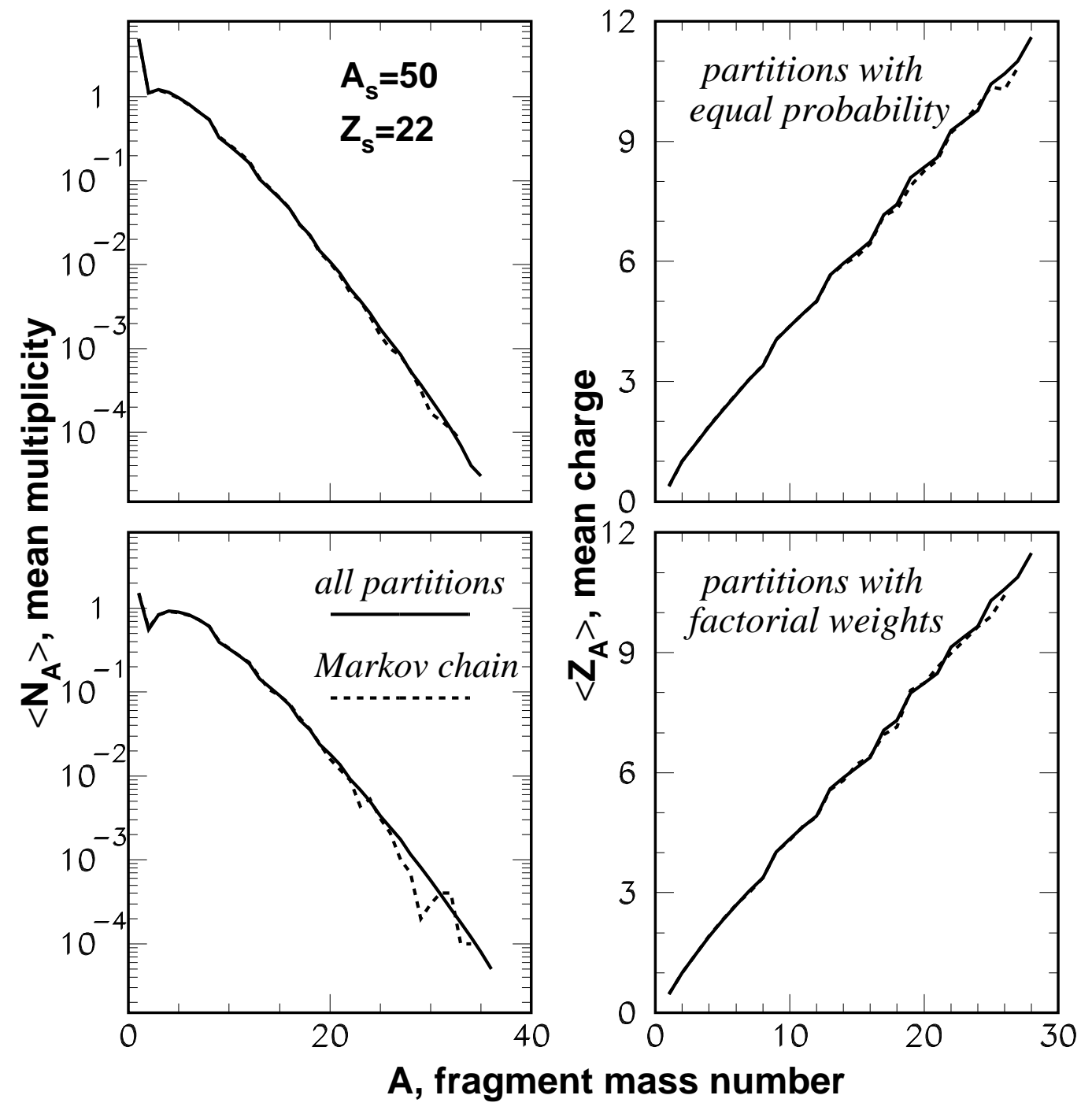

FIG. 1. Mean multiplicities and charges of fragments obtained by the Markov chain generation of partitions (dashed lines) as compared with direct accounting for all partitions (solid lines), for the system with total mass number $A_{s}=50$ and charge $Z_{s}=22$. In the top panels partitions are taken with equal probability, in the bottom ones partitions have factorial weights. 
the multiplicity of fragments with mass number $A$ and charge $Z$. Despite of the fact that these weights are essentially different, the results obtained in the direct and Markov chain calculations coincide in both cases. The same good agreement holds for other weights and fragment partition characteristics.

In the beginning we consider simple thermal effects concerning isospin distribution in multifragmentation. In grand canonical limit the formulae for the average charge $\left\langle Z_{A}\right\rangle$ of fragment $A$ and the width $\sigma_{Z}^{A}$ of the charge distribution were obtained in $8 \rrbracket$ :

$$
\left\langle Z_{A}\right\rangle=\frac{(4 \gamma+\nu) A}{8 \gamma+2 c A^{2 / 3}}, \quad \sigma_{Z}^{A} \approx \sqrt{\frac{A T}{8 \gamma}},
$$

where $T$ is the temperature, $\gamma \approx 25 \mathrm{MeV}$ is the symmetry energy coefficient, $\nu$ is the chemical potential responsible for the charge conservation. A Coulomb energy of the system is accounted in the mean-field Wigner-Seitz approximation leading to $c=(3 / 5)\left(e^{2} / r_{0}\right)(1-$ $\left.\left(\rho / \rho_{0}\right)^{1 / 3}\right)$, where $e$ is the proton charge, $r_{0}=1.17 \mathrm{fm}, \rho$ is the nuclear freeze-out density and $\rho_{0} \approx 0.15 \mathrm{fm}^{-3}$ is the normal nuclear density. The $A$-dependence of $\left\langle Z_{A}\right\rangle$ recalls a famous experimental systematics for the stable nuclei [13]: $\left\langle Z_{\text {stable }}\right\rangle=A /\left(1.98+0.0155 A^{2 / 3}\right)$. The difference is in the additional chemical potential $\nu$ and in the reduced Coulomb coefficient. As calculations show, $\nu$ decreases with the neutron excess of the source so that the fragments produced from a neutron-rich source are also neutron-rich (see e.g. Fig. 3 in [14]).

In finite systems isospin of the fragments changes with the excitation energy in a special way. In Fig. 2 we show neutron-to-proton ratios $(\mathrm{N} / \mathrm{Z})$ of the fragments together with their mass distributions. The calculations were done with the Markov chain SMM for Au source $\left(A_{s}=197, Z_{s}=79\right)$ at density $\rho_{s}=\rho_{0} / 3$ using the Wigner-Seitz approximation as in previous standard SMM calculations [14]. However, the direct evaluation of the Coulomb interaction does not change the presented results. One can see a general statistical trend: the N/Z ratio of hot primary fragments increases with their mass numbers, as is also evident from formula (1). This is a consequence of the interplay between Coulomb and symmetry energy contributions to the binding energy of fragments [2]. This trend persists up to $A \leq A_{s} / 2$, at larger $A$ the finite-size effects due to the mass and charge conservation demolish it. In Fig. 2 

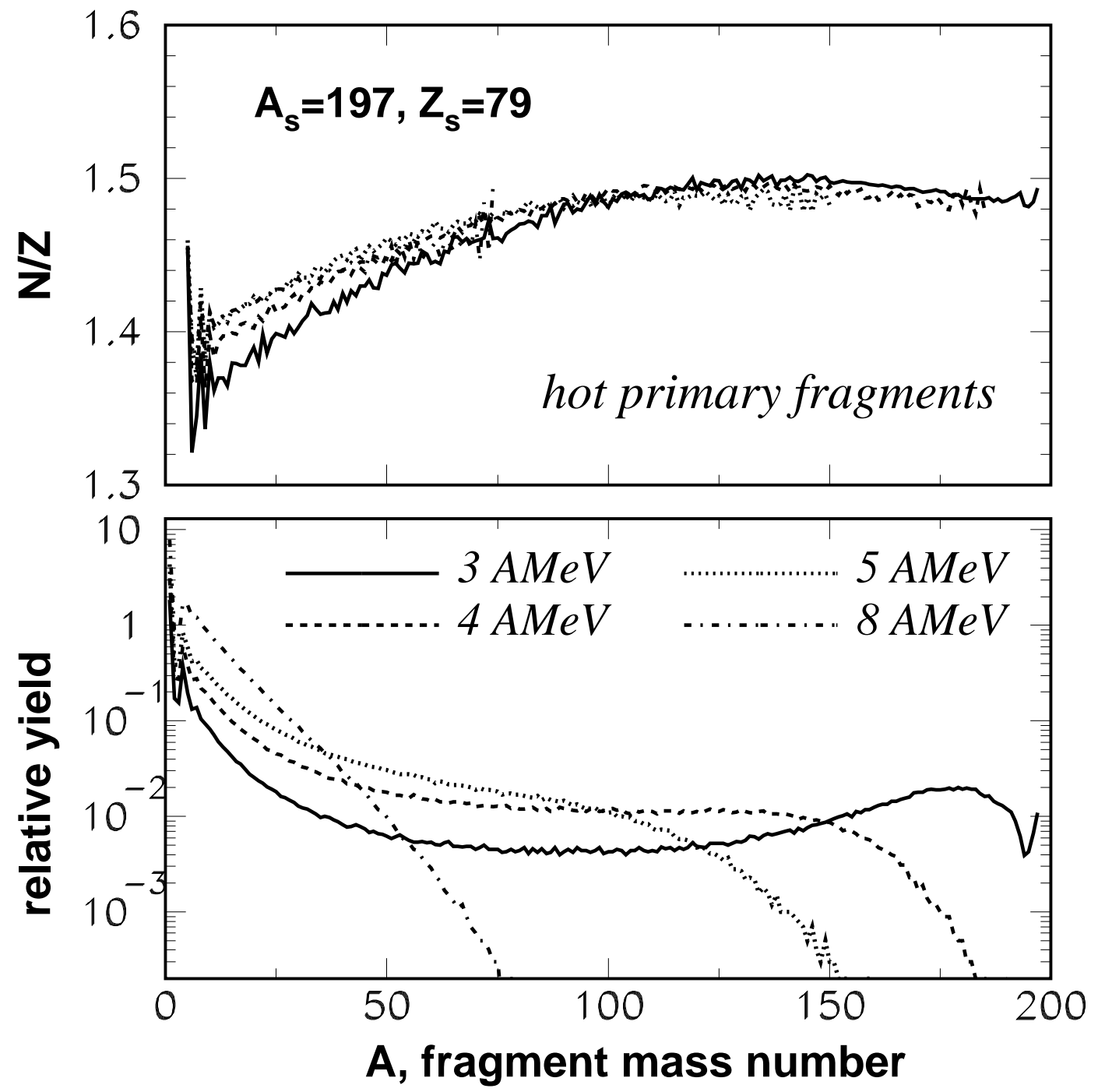

FIG. 2. The neutron-to-proton ratio $\mathrm{N} / \mathrm{Z}$ and relative yield of hot primary fragments produced after break-up of Au nucleus at different excitation energies: 3 (solid lines), 4 (dashed lines), 5 (dotted lines) and 8 (dot-dashed lines) $\mathrm{MeV}$ per nucleon. 
we demonstrate also the evolution of $\mathrm{N} / \mathrm{Z}$ ratio and mass distribution of fragments in the excitation energy range $E_{s}^{*}=3-8 \mathrm{MeV} /$ nucleon. It is seen that the fragment mass distribution evolves from the U-shape, at the multifragmentation threshold $E_{s}^{*} \sim 3 \mathrm{MeV} /$ nucleon, to an exponential fall at high energies. As the energy increases the $\mathrm{N} / \mathrm{Z}$ ratio of primary intermediate mass fragments (IMF, charges $\mathrm{Z}=3-20$ ) increases too. The reason is that the heaviest neutron-rich fragments are destroyed at increasing excitation energy and their neutrons are combined into the IMFs, since the number of free neutrons is still small at this stage. Simultaneously the N/Z ratio of the heaviest fragments decreases slightly. At very high excitation energy $\left(E_{s}^{*}>8 \mathrm{MeV} /\right.$ nucleon $)$ the $\mathrm{N} / \mathrm{Z}$ ratio of IMFs does not rise anymore but drops too because no heavier fragments are left and the number of free neutrons increases fastly.

We note that this isospin evolution takes place in the energy range which is usually associated with a liquid-gas type phase transition in finite nuclei [2,5]. Our calculations show how the isospin fractionation phenomenon [15] associated with this phase transition actually shows up in finite nuclear systems. This effect of a "rise-and-fall" of the IMF's neutron content (see also Fig. 4 in [14]) is sensitive to the details of the description of hot primary fragments (e.g. binding energy, shell effects) as well as to the size and isospin content of the thermal source. Therefore, it is a good tool for probing the freeze-out conditions. Moreover, this effect can help to reveal the difference between dynamical and statistical mechanisms of multifragmentation. As was shown in many papers (e.g. [16, 17]), both approaches give nearly similar results concerning the IMF yields. However, the dynamical models favor the opposite isospin effect by decreasing the $\mathrm{N} / \mathrm{Z}$ ratio of larger fragments and thus making them more symmetric [17,18]. In such a case the dynamics provides a decreasing neutron content of IMFs for larger excitation energies of sources (see e.g. [18]) contrary to the experimental observation 14 .

It is instructive to see how an angular momentum influences the isospin of fragments emitted from a single source. In this calculation we assume that the total excitation energy and angular momentum are fixed and that the sharing between rotational and thermal 

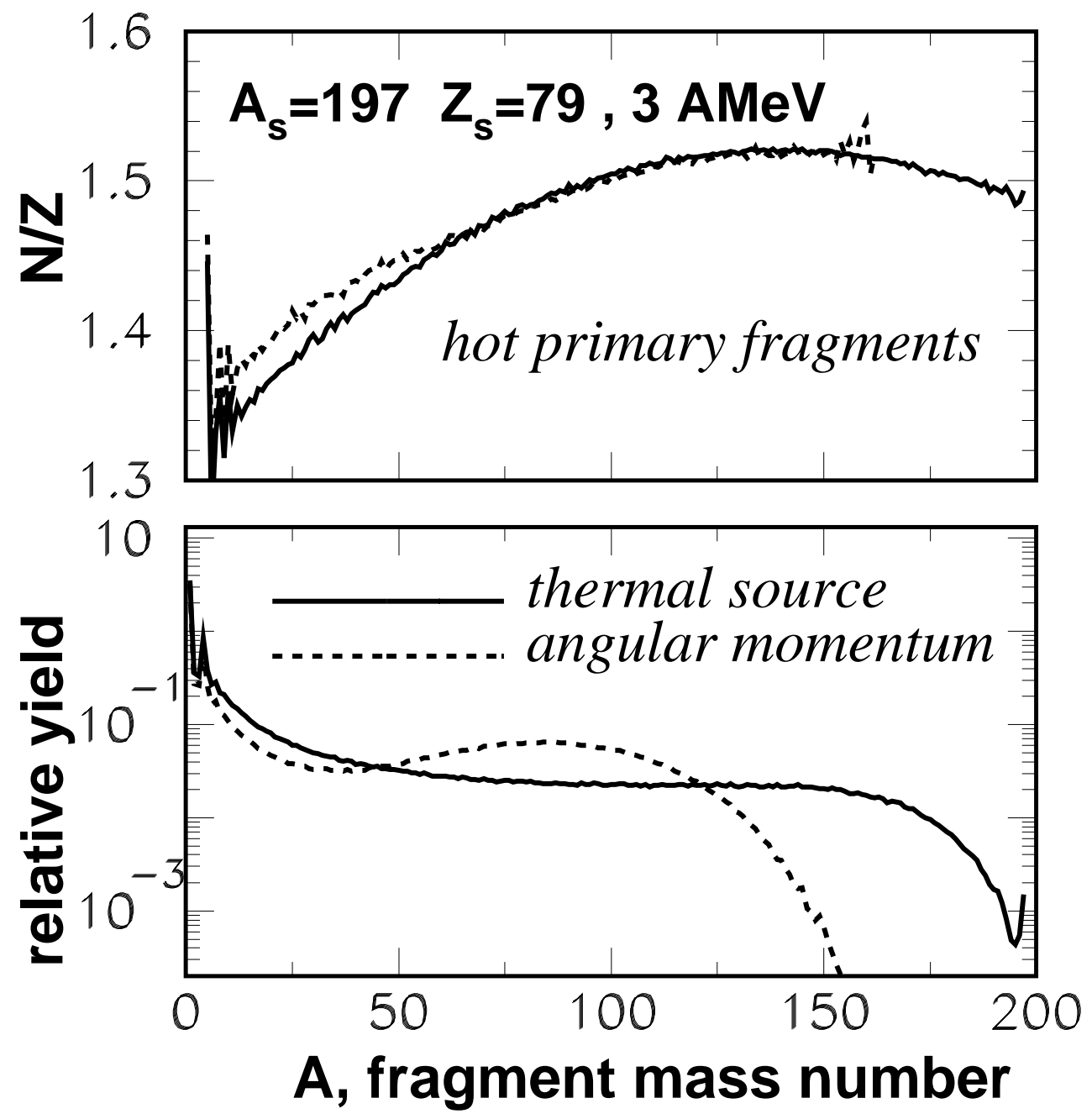

FIG. 3. The neutron-to-proton ratio $\mathrm{N} / \mathrm{Z}$ and relative yield of hot primary fragments produced after break-up of Au nucleus. Solid lines: Markov chain SMM calculations for a thermal source with excitation energy $3 \mathrm{MeV} /$ nucleon, dashed lines: the same source with angular momentum $150 \hbar$. 
energies is governed by the statistical weights of different configurations. In Fig. 3 we show yields and $\mathrm{N} / \mathrm{Z}$ ratios of hot primary fragments produced in the freeze-out volume after break-up of $\mathrm{Au}$ source at $\rho_{s}=\rho_{0} / 6$ and $E_{s}^{*}=3 \mathrm{MeV}$ per nucleon. It is seen that an angular momentum favors fission-like fragment partitions with two large equal-size fragments (see also Refs. [3, 12]). That is different from a normal fragmentation pattern dominated by partitions with different-size fragments. An angular momentum leads to an increasing $\mathrm{N} / \mathrm{Z}$ ratio of IMFs as well. The latter effect is important and has a simple qualitative explanation. An angular momentum favors the emission of IMFs with larger mass numbers, providing a larger moment of inertia, in oder to minimize the rotational energy and maximize the entropy. On the other side the Coulomb interaction prevents the emission of IMFs with large charges. As a result of interplay of these two factors we obtain the increasing $\mathrm{N} / \mathrm{Z}$ ratio.

In peripheral nucleus-nucleus collisions at the projectile energies of $10-100 \mathrm{MeV} /$ nucleon the break-up of highly excited projectiles-like nuclei is fast (the characteristic time is around $100 \mathrm{fm} / \mathrm{c}$ ) and happens in the vicinity of a target-like residue. The Coulomb field of the target residue influences the fragmentation of the projectile source by increasing the charge asymmetry of produced fragments and leading to a non-isotropic fragment emission: small fragments are preferably emitted to the side of the target [19]. Within the Markov chain SMM one can study how this effect influences the isotope composition of fragments.

Calculations were performed for the same Au source as in Fig. 3. The source was placed at a fixed distance $(20 \mathrm{fm})$ from another Au source simulating the target residue. This distance was obtained under assumption that the break-up happens at $\sim 100 \mathrm{fm} / \mathrm{c}$ after a peripheral collision of $35 A \cdot \mathrm{MeV} \mathrm{Au}$ projectile with $\mathrm{Au}$ target. In reality the break-up may happen at various distances, excitation energies and angular momenta. In statistical approach we can take into account the distribution of sources in distances and other characteristics by considering an ensemble of the sources. Parameters of this ensemble can be found by global comparison with the experiment [2,20]. However, the approximation of a fixed distance is sufficient for the qualitative identification of a new statistical effect. 

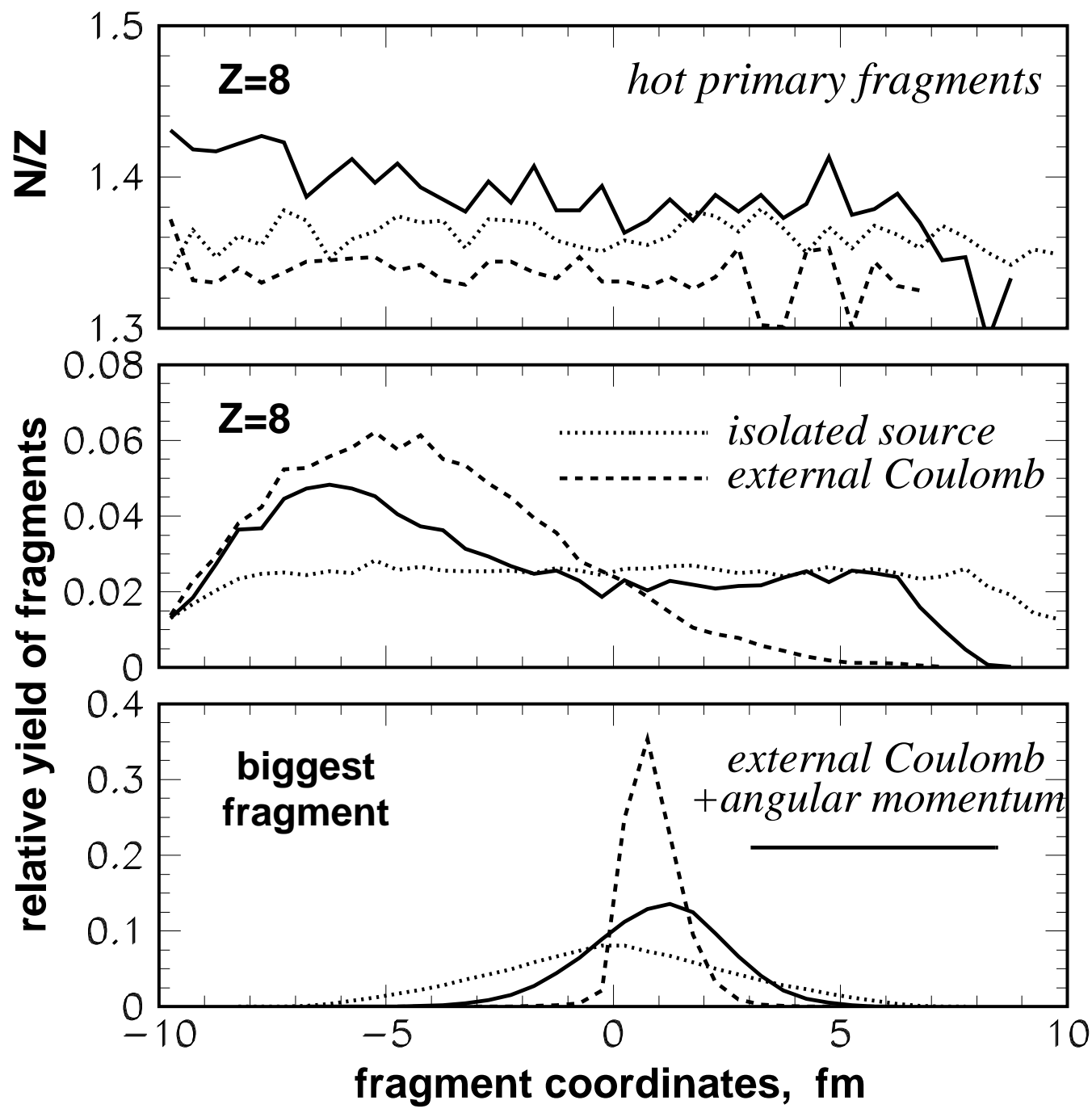

FIG. 4. Freeze-out coordinate distributions of neutron-to-proton ratio N/Z of primary fragments with $\mathrm{Z}=8$ (top panel) and relative yields of the primary $\mathrm{Z}=8$ and biggest fragments (middle and bottom panels) produced at break-up of Au source at excitation energy of $3 \mathrm{MeV} / \mathrm{nucleon}$. The second Au nucleus is placed at $-20 \mathrm{fm}$ from the center of the freeze-out volume. Dotted lines: the isolated Au source, dashed lines: Coulomb interaction with the second Au nucleus is included, solid lines: angular momentum of $150 \hbar$ is included as well. 
In Fig. 4 we show the spatial distributions of yields and N/Z ratios of hot primary IMFs with $Z=8$ and the biggest fragments in the freeze-out volume along the axis connecting the projectile and target sources. It is seen that in the case of a single isolated source all distributions are symmetric with respect to the center of mass of the source. In the case of an external Coulomb field induced by the target source, the IMFs are mainly located at the target side while the biggest fragments are shifted to the opposite direction. Such positioning of fragments minimizes the Coulomb energy of the target-projectile system. However, the external Coulomb field alone cannot affect essentially the fragment isospin distribution. In case of an additional angular momentum the $\mathrm{N} / \mathrm{Z}$ ratio of the IMFs increases considerably and becomes larger for the IMFs which are closer to the target. The reason is again an interplay between the Coulomb and rotational energy. To maximize the moment of inertia the system needs more heavy IMFs, while to minimize the Coulomb energy, depending on the fragment distance to the target, the IMFs should have smaller charges.

Our calculations show that this asymmetry of the IMF isospin distribution survives after secondary deexcitation of hot fragments. The following Coulomb propagation pushes the IMFs in the direction of the target providing predominant population of the midrapidity kinematic region by neutron-rich fragments. The Coulomb repulsion from the sources may be not sufficient to accelerate fragments up to the energy corresponding to the center of mass velocity of the colliding nuclei. Nevertheless, the IMFs can populate a considerable part of the midrapidity region [19]. Within the statistical picture a slight radial flow can supply the IMFs with additional velocities to fill the central part of the midrapidity zone. Such mechanism of the neutron-rich IMF production is consistent with the experimental data [21,22].

In conclusion, a new Markov chain version of the SMM was applied for analysis of the isotope composition of fragments and its evolution. The increasing $\mathrm{N} / \mathrm{Z}$ ratio of IMFs with excitation energy can be considered as a manifestation of the isospin fractionation associated with the phase transition in finite nuclei. It was also shown that in peripheral nucleus-nucleus collisions the characteristics of statistically produced fragments depend on 
Coulomb interaction between the target- and projectile-like sources and on angular momentum transferred to the sources. In particular, this leads to spatial asymmetry of both the fragment emission and their isotope composition respective to the source center of mass. Previously the symmetry violation was considered as a sign of the dynamical emission from the neck region. However, we have demonstrated that there exists an alternative statistical explanation: the symmetry of the phase space population is broken by the interaction between two sources. Theoretically such a process gives example of a new kind of statistical emission influenced by an inhomogeneous external long-range field [19.

A.S.B. thanks GSI for financial support and warm hospitality. I.N.M. thanks the Humboldt Foundation for the financial support. 


\section{REFERENCES}

[1] See for instance: "Multifragmentation", Proceedings of the International Workshop 27 on Gross Properties of Nuclei and Nuclear Excitations, Hirschegg, Austria, January 17-23, 1999. GSI, Darmstadt, 1999.

[2] J.P.Bondorf, A.S.Botvina, A.S.Iljinov, I.N.Mishustin and K.Sneppen. Phys. Rep. 257, $133(1995)$.

[3] D.H.E.Gross, Phys.Rep. 279, 119 (1997).

[4] A.S.Botvina et al., Nucl.Phys. A584 (1995) 737.

[5] M.D'Agostino et al., Nucl. Phys. A650, 329 (1999).

[6] M.D'Agostino et al., Phys. Lett. B371, 175 (1996).

[7] C.Williams et al. Phys. Rev. C55, R2132 (1997).

[8] A.S.Botvina et al., Nucl.Phys. A475 (1987) 663.

[9] A.S.Botvina, A.D.Jackson and I.N.Mishustin, Phys. Rev. E62, R64 (2000).

[10] A.S.Botvina, I.N.Mishustin et al., in preparation.

[11] K.L. Chung, Markov processes with stationary transition probability. Springer, Heidelberg, 1967.

[12] A.S.Botvina and D.H.E.Gross, Nucl. Phys. A592, 257 (1995).

[13] P.Marmier and E.Sheldon, Physics of Nuclei and Particles. Academic Press, N.Y., 1969

[14] P.M.Milazzo, A.S.Botvina, G.Vannini et al., Phys. Rev. C62, 041602(R) (2000).

[15] H.Müller and B.D.Serot, Phys. Rev. C52, 2072 (1995)

[16] R.Nebauer, J.Aichelin et al., Nucl. Phys. A658, 67 (1999).

[17] A.B.Larionov, A.S.Botvina, M.Colonna and M.Di Toro, Nucl. Phys. A658, 375 (1999). 
[18] M.Di Toro et al., nucl-th/0009079, 2000.

[19] A.S.Botvina, M.Bruno, M.D'Agostino and D.H.E.Gross, Phys. Rev. C59, 3444 (1999).

[20] P.Desesquelles et al., Nucl. Phys. A633, 547 (1998).

[21] J.F.Dempsey et al., Phys. Rev. C54, 1710 (1996).

[22] Y.Larochelle et al., Phys. Rev. C62, 051602(R) (2000). 